\title{
The Radiation Resistance of End-Fire and Collinear Arrays ${ }^{*}$
}

\author{
CHARLES H. PAPAS†, ASSOciate, I.R.E., AND RONOLD KING $\dagger$, SENIOR MEMBER, I.R.E.
}

Summary.-Expressions for the radiation resistances of end-fire and collinear arrays of half-wave dipoles are obtained in terms of circular functions in a form convenient for computation. No mathematical approximations except for a Fourier representation ${ }^{1}$ of the field of a single half-wave dipole are used. The first integral theorem of Sonine $e^{2}$ and an integral representation of the Bessel function due to Hansen ${ }^{3}$ are involved in the integration of the normal component of Poynting's vector.

Results computed from the new formula for the radiation resistance of an $n$-element parallel array in which the spacings and successive phasings of the dipole elements are 180 degrees (bilateral end-fire) agree closely with those of Pistolkors, ${ }^{4}$ who used Brillouin's e.m.f. method; they are a little less than the figures of BontschBruewitsch, ${ }^{5}$ who numerically integrated Poynting's vector. Calculations for the radiation resistance of an $n$-element collinear array using the new formula are compared with those of Bontsch-Bruewitsch, with which they are in satisfactory agreement. The new formula is also used to compute the radiation resistance of an $n$-element unilateral end-fire array (i.e., an $n$-element parallel array in which the spacings and successive phasings of the dipole elements are 90 degrees).

\section{Introduction and COMPARISON OF Results}

$\prod^{N}$ $\mathrm{N}$ HIS PATENT Campbell ${ }^{6}$ has calculated and plotted the radiation patterns of rectilinear arrays composed of 16 elements for spacings from 0 to 4 wavelengths in steps of one-eighth wavelength and successive phasings from 0 to one-half period in steps of one-eighth period. From these patterns it is seen that, when the elements are spaced one-half wavelength apart and successively phased one-half period, a bilateral end-fire is obtained. By reducing the spacing to one-quarter wavelength and the phasing to onequarter period, a unilateral end-fire is obtained.

The radiation resistance of bilateral end-fire arrays has been calculated by Pistolkors ${ }^{4}$ using the e.m.f. method for arrays having $2,3,4,5,6$, and 7 elements. Bontsch-Bruewitsch ${ }^{5}$ has calculated the radiation re-

* Decimal classification: R325.113 $\times$ R221. Original manuscript received by the Institute, January 21,1948 . The research reported in this document was made possible through support extended Cruft Laboratory, Harvard University, jointly by the Navy Department (Office of Naval Research) and the Signal Corps, U. S. Army, under Contract N5ori-76, T.O.I.

† Cruft Laboratory, Harvard University, Cambridge, Mass.

1 Ronold W. P. King, "The approximate representation of the distant field of linear radiators," Proc. I.R.E., vol. 29, pp. 458-464; August, 1941.

${ }^{2}$ N. J. Sonine, "Recherches sur les fonctions cylindriques et le development des fonctions continues en series," Math. Ann., vol. 16, pp. $1-80 ; 1880$.

"P. A. Hansen, in Jahnke and Emde, "Tables of Functions," p. 149, Dover Publications, New York, N. Y.; 1943.

- A. A. Pistolkors, "The radiation resistance of beam antennas," Proc. I.R.E., vol. 17, pp. 562-579; March, 1929.

b M. A. Bontsch-Bruewitsch, "Die Strahlung der komplizierten rechtwinkeligen antennen mit gleichbeschaffenen Vibratoren," Ann. Physik., vol. 81, pp. 425-453; 1926.

${ }^{6}$ G. A. Campbell, U. S. Patent No. 1, 783, 522. sistances of bilateral arrays by numerically integrating Poynting's vector for arrays consisting of $2,3,4$, and an infinite number of elements. Table I shows the results of Pistolkors $(P)$, Bontsch-Bruewitsch $(B B)$, and those of this paper $(P K)$, the last named computed from (26).

TABLE I

Average Radiation Resistance* of Bilateral Array

\begin{tabular}{cccl}
\hline $\begin{array}{c}\text { Number of } \\
\text { Elements }\end{array}$ & $P$ & $B B$ & $P K$ \\
\hline 2 & 85.7 ohms & 88 ohms & 82.30 ohms \\
3 & 92.5 & 95 & 87.72 \\
4 & 96.8 & 100 & 91.04 \\
5 & 99.8 & & 93.30 \\
6 & 102.1 & & 94.95 \\
7 & 103.9 & 120 & 96.22 \\
Infinite & & & \\
\hline
\end{tabular}

* Average radiation resistance means the total radiation resistance divided by the number of elements: $\left(R_{0} \% / n\right)$.

The radiation resistance of $n$ collinear dipoles has been calculated by Bontsch-Bruewitsch, ${ }^{5}$ who obtained the following formula:

$R=(-1)^{n-1} 60\left[\begin{array}{ll}n \pi & (n-1) \pi \\ S-4 & S \\ 0 & 0\end{array}+\underset{c}{(n-2) \pi} \quad \cdots\right]$

where

$$
S_{0}^{n \pi}=\int_{0}^{m \pi} \frac{\sin ^{2} \mu}{\mu} d \mu
$$

The results obtained from (1) for $n=1,2,3,4$, and 5 and from the new formula (38) are shown in Table II.

TABLE II

Average Radiation Resistance* of Collinear Arrays

\begin{tabular}{ccc}
\hline $\begin{array}{c}\text { Number of } \\
\text { elements }\end{array}$ & $B B$ & $P K$ \\
\hline$n=1$ & 98 ohms & 71.44 ohms \\
2 & 103 & 93.15 \\
3 & 106 & 96.77 \\
4 & 108 & 101.78 \\
5 & & 102.05 \\
6 & & 102.82 \\
7 & & \\
\hline
\end{tabular}

* Average radiation resistance means the total radiation resistance divided by the number of elements: $\left(R_{0}^{\circ} / n\right)$.

The radiation resistances of unilateral end-fire arrays computed from (26) for spacings and phasings of $90^{\circ}$ are shown in Table III. 
TABLE III

Average Radiation Resistance* of Unilateral END-FIRE ARRAYS

\begin{tabular}{cl}
\hline $\begin{array}{c}\text { Number of } \\
\text { elements }\end{array}$ & $P K$ \\
\hline 2 & 71.44 ohms \\
3 & 78.68 \\
4 & 82.30 \\
5 & 85.55 \\
6 & 87.72 \\
7 & 89.62 \\
\hline
\end{tabular}

* Average radiation resistance means the total radiation resistance divided by the number of elements: $\left(R_{0} / n\right)$.

\section{Calculation of the Radiation Resistance oF END-Fire ARRAYS}

In the derivation of the new formulas for the radiation resistance, it is tacitly assumed that changes in spacing and phasing between the dipoles have a negligible effect upon the current distribution in the individual dipoles. This is a good approximation for center-driven half-wave dipoles for all spacings. (It is a poor approximation for full-wave dipoles.)

The total radiated power is obtained by integrating the normal component of the Poynting vector $S$ over a large sphere of radius $R$. If $W$ is the total radiated power in watts,

$$
\begin{aligned}
W & =\int_{0}^{2 \pi} \int_{0}^{\pi} S R^{2} \sin \theta \cdot d \theta \cdot d \phi \\
& =\frac{30 I_{0}{ }^{2}}{2 \pi} \int_{0}^{2 \pi} \int_{0}^{\pi} V^{2}(\theta) \cdot A^{2}(\theta, \phi) \cdot \sin \theta \cdot d \theta \cdot d \phi
\end{aligned}
$$

where $V(\theta)$ is the field factor of a single dipole, $A(\theta, \phi)$ is the array factor, and $I_{0}$ is the current amplitude at a loop position. They are contained explicitly in the following formula for the Poynting vector:

$$
\begin{aligned}
S=\frac{30 I_{0}^{2}}{2 \pi R^{2}}\left\{\frac{\cos \left(\frac{\pi}{2} \cdot \cos \theta\right)}{\sin \theta}\right. & \\
& \left.\frac{\sin n\left(\pi \frac{s}{\lambda} \sin \theta \cos \phi+\pi \frac{s}{\lambda}\right)}{\sin \left(\pi \frac{s}{\lambda} \sin \theta \cos \phi+\pi \frac{s}{\lambda}\right)}\right\}^{2} .
\end{aligned}
$$

In this expression, $n$ is the number of half-wave dipoles in the array, $s$ is the uniform spacing of the radiators, $R$ is the radial distance from the center of the array to the point of calculation, and $\theta$ and $\phi$ are the polar and azimuthal angles, respectively. The quantity in brackets is the product of the two functions $V(\theta)$ and $A(\theta, \phi)$.

$$
V(\theta) \equiv \frac{\cos \left(\frac{\pi}{2} \cos \theta\right)}{\sin \theta}, \quad A(\theta, \phi) \equiv \frac{\sin n y}{\sin y}
$$

where

$$
y=\pi \frac{s}{\lambda} \sin \theta \cdot \cos \phi+\pi \frac{s}{\lambda} \equiv K_{1} \cos \phi+K_{2} .
$$

In order to integrate the square of the array factor, it is expanded in a series of cosine terms and integrated term by term. Since the series contains only a finite number of terms, no difficulty arises in the term-byterm integration.

$$
A^{2}(\theta, \phi)=\frac{\sin ^{2} n y}{\sin ^{2} y}=n+\sum_{r=2}^{2 n-2}(2 n-r) \cos r y
$$

where $r=2,4,6,8, \cdots$ even integer. Now, integrate both sides of $(7)$ with respect to $\phi$ :

$$
\begin{aligned}
\int_{0}^{2 \pi} & A^{2}(\theta, \phi) d \phi \\
& =\int_{0}^{2 \pi} n d \phi+\sum_{r=2}^{2 n-2}(2 n-r) \int_{0}^{2 \pi} \cos (r y) \cdot d \phi
\end{aligned}
$$

Hansen' ${ }^{3}$ integral representation of a Bessel function is

$$
\begin{aligned}
\int_{0}^{2 \pi} \cos (r y) d \phi & =\int_{0}^{2 \pi} \cos r\left(K_{1} \cos \phi+K_{2}\right) d \phi \\
& =2 J_{0}\left(r K_{1}\right) \cos \left(r K_{2}\right) .
\end{aligned}
$$

Therefore, the integral of (7) with respect to $\phi$ is

$$
\begin{aligned}
\int_{0}^{2 \pi} A^{2}(\theta, \phi) d \phi= & 2 \pi n+\sum_{r=2}^{2 n-2}(2 n-r) \\
& \cdot 2 \pi J_{0}\left(r K_{1}\right) \cos \left(r K_{2}\right)=H(\theta) .
\end{aligned}
$$

Note that each term on the right is a function only of the polar angle $\theta$. It is represented by $H(\theta)$.

The integral representation of $W$ in (3) now becomes

$$
W=\frac{30 I_{0}^{2}}{2 \pi} \int_{0}^{\pi} V^{2}(\theta) \cdot H(\theta) \sin \theta d \theta
$$

Introduction of the Fourier approximation ${ }^{1}$

$$
V(\theta) \doteq 0.945 \sin \theta
$$

in (11) gives

$$
W=\frac{30 I_{0}^{2}}{2 \pi} \int_{0}^{\pi}(0.945)^{2} \cdot H(\theta) \sin ^{3} \theta \cdot d \theta .
$$

$H(\theta) \sin ^{3} \theta$ is a finite series, each term of which must be integrated with respect to $\theta$ :

$$
\begin{aligned}
& \int_{0}^{\pi} H(\theta) \sin ^{3} \theta d \theta=2 \pi n \int_{0}^{\pi} \sin ^{3} \theta d \theta \\
& +2 \pi \sum_{r=2}^{2 n-2}(2 n-r) \cdot \cos \left(r K_{2}\right) \int_{0}^{\pi} J_{0}\left(r K_{1}\right) \sin ^{3} \theta d \theta .
\end{aligned}
$$

The next task is to evaluate the integral

$$
\int_{0}^{\pi} J_{0}\left(r K_{1}\right) \sin ^{3} \theta \cdot d \theta
$$


From symmetry considerations of the integrand, this may be rewritten in the following way:

$$
\begin{aligned}
\int_{0}^{\pi} J_{0}\left(r K_{1}\right) & \sin ^{3} \theta \cdot d \theta \\
= & 2 \int_{0}^{\pi / 2} J_{0}\left(r \pi \frac{s}{\lambda} \sin \theta\right) \sin ^{3} \theta \cdot d \theta .
\end{aligned}
$$

Since $\sin ^{2} \theta=1-\cos ^{2} \theta$, the integral (15) is the difference of two integrals of which each is in Sonine ${ }^{2}$ form. Sonine's first integral ${ }^{2}$ is given by

$$
\begin{aligned}
\int_{0}^{\pi / 2} J_{\nu}(Z \sin \theta) \cdot \cos ^{2 \mu+1} \theta \cdot \sin ^{\nu+1} \theta d \theta & \\
= & 2^{\mu} \Gamma(\mu+1) \frac{J_{\nu+\mu+1}(Z)}{Z^{\mu+1}} .
\end{aligned}
$$

With $\mu=-\frac{1}{2}$ and $\nu=0$ and substituting $r \pi / s \lambda$ for $Z$, the result is

$$
\int_{0}^{\pi / 2} J_{0}\left(r \pi \frac{s}{\lambda} \sin \theta\right) \sin \theta d \theta=\frac{\Gamma\left(\frac{1}{2}\right) J_{1 / 2}\left(r \pi \frac{s}{\lambda}\right)}{\sqrt{2} \sqrt{r \pi \frac{s}{\lambda}}} .
$$

Similarly, if with $\mu=\frac{1}{2}$ and $\nu=0$ and substituting $r \pi(s / \lambda)$ for $Z$,

$$
\begin{aligned}
\int_{0}^{x / 2} J_{0}\left(r \pi \frac{s}{\lambda} \sin \theta\right) \cos ^{2} \theta \cdot \sin \theta \cdot d \theta \\
=\frac{\sqrt{2} \Gamma\left(\frac{8}{2}\right) J_{3 / 2}\left(r \pi \frac{s}{\lambda}\right)}{\left(r \pi \frac{s}{\lambda}\right)^{3 / 2}},
\end{aligned}
$$

therefore,

$$
\begin{aligned}
& 2 \int_{0}^{\pi / 2} J_{0}\left(r \pi \frac{s}{\lambda} \sin \theta\right) \sin ^{3} \theta \cdot d \theta \\
& =\frac{2 \Gamma\left(\frac{1}{2}\right) J_{1 / 2}\left(r \pi \frac{s}{\lambda}\right)}{\sqrt{2} \sqrt{r \pi \frac{s}{\lambda}}}-\frac{2 \sqrt{2} \Gamma\left(\frac{3}{2}\right) J_{3 / 2}\left(r \pi \frac{s}{\lambda}\right)}{\left(r \pi \frac{s}{\lambda}\right)^{3 / 2}} .
\end{aligned}
$$

The expression for the radiated power then takes the form:

$$
\begin{gathered}
W=\frac{30 I_{0}^{2}}{2 \pi}(0.945)^{2}\left[\frac{8 \pi n}{3}+2 \pi \sum_{r=2}^{2 n-2}(2 n-r) \cdot \cos \left(r K_{2}\right)\right. \\
\left\{\begin{array}{c}
\frac{\sqrt{2} \Gamma\left(\frac{1}{2}\right) J_{1 / 2}\left(r \pi \frac{s}{\lambda}\right)}{\sqrt{r \pi \frac{s}{\lambda}}} \\
\left.\left.-\frac{2 \sqrt{2} \Gamma\left(\frac{3}{2}\right) J_{3 / 2}\left(r \pi \frac{s}{\lambda}\right)}{\left(r \pi \frac{s}{\lambda}\right)^{3 / 2}}\right\}\right]
\end{array}\right.
\end{gathered}
$$

and the external or radiation resistance $R_{0}{ }^{e}=2 W / I_{0}{ }^{2}$ becomes

$$
\begin{array}{r}
R_{0}{ }^{e}=\frac{60}{2 \pi}(0.945)^{2}\left(\frac{8 \pi}{3} n+2 \pi \sum_{r=2}^{2 n-2}(2 n-r) \cos \left(r K_{2}\right)\right. \\
\cdot\left\{\begin{array}{c}
\frac{\sqrt{2} \Gamma\left(\frac{1}{2}\right) J_{1 / 2}\left(r \pi \frac{s}{\lambda}\right)}{\sqrt{r \pi \frac{s}{\lambda}}} \\
\left.-\frac{2 \sqrt{2} \Gamma\left(\frac{3}{2}\right) J_{3 / 2}\left(r \pi \frac{s}{\lambda}\right)}{\left(r \pi \frac{s}{\lambda}\right)^{3 / 2}}\right\}
\end{array}\right.
\end{array}
$$

This expression involves Bessel functions with fractional indexes which are not convenient for computation. It is possible to transform them into circular functions using

$$
J_{1 / 2}\left(r \pi \frac{s}{\lambda}\right)=\sqrt{\frac{2}{\pi}} \frac{\sin \left(r \pi \frac{s}{\lambda}\right)}{\sqrt{r \pi \frac{s}{\lambda}}}
$$

and

$$
\begin{aligned}
& J_{3 / 2}\left(r \pi \frac{s}{\lambda}\right) \\
& =\sqrt{\frac{2}{\pi}}\left\{\frac{\sin \left(r \pi \frac{s}{\lambda}\right)}{\left(r \pi \frac{s}{\lambda}\right)^{3 / 2}}-\frac{\cos \left(r \pi \frac{s}{\lambda}\right)}{\sqrt{r \pi \frac{s}{\lambda}}}\right\} .
\end{aligned}
$$

Evidently,

$$
\frac{J_{1 / 2}\left(r \pi \frac{s}{\lambda}\right)}{\sqrt{r \pi \frac{s}{\lambda}}}=\sqrt{\frac{2}{\pi}} \frac{\sin \left(r \pi \frac{s}{\lambda}\right)}{r \pi \frac{s}{\lambda}},
$$

and

$$
\begin{aligned}
& \frac{J_{3 / 2}\left(r \pi \frac{s}{\lambda}\right)}{\left(r \pi \frac{s}{\lambda}\right)^{3 / 2}} \\
& \quad=\sqrt{\frac{2}{\pi}}\left\{\frac{\sin \left(r \pi \frac{s}{\lambda}\right)}{\left(r \pi \frac{s}{\lambda}\right)^{3}}-\frac{\cos \left(r \pi \frac{s}{\lambda}\right)}{\left(r \pi \frac{s}{\lambda}\right)^{2}}\right\} .
\end{aligned}
$$

Since $\Gamma\left(\frac{1}{2}\right)=\sqrt{\pi}$ and $\Gamma(3 / 2)=\frac{1}{2} \sqrt{\pi}$, the final expression for the radiation resistance is 


$$
\begin{aligned}
R_{0^{*}}= & \frac{60}{2 \pi}(0.945)^{2}\left[\frac{8 \pi n}{3}+4 \pi \sum_{r=2}^{2 n-2}(2 n-r)\right. \\
& \cdot \cos \left(r K_{2}\right) \Lambda\left(r \pi \frac{s}{\lambda}\right)
\end{aligned}
$$

where

$$
\begin{aligned}
\Lambda\left(r \pi \frac{s}{\lambda}\right)= & \frac{\sin \left(r \pi \frac{s}{\lambda}\right)}{r \pi \frac{s}{\lambda}}-\frac{\sin \left(r \pi \frac{s}{\lambda}\right)}{\left(r \pi \frac{s}{\lambda}\right)^{3}} \\
& +\frac{\cos \left(r \pi \frac{s}{\lambda}\right)}{\left(r \pi \frac{s}{\lambda}\right)^{2}} .
\end{aligned}
$$

This formula is readily evaluated numerically. The results are shown in Tables I, III, and IV.

\section{Calculation of the Radiation Resistance of Collinear Arrays}

The radiation resistance of an $n$-element collinear antenna is given by the well-known integral:

$R_{0^{*}}=60 \int_{0}^{\pi} \frac{\cos ^{2}\left(\frac{\pi}{2} \cos \theta\right)}{\sin ^{2} \theta} \cdot \frac{\sin ^{2} n\left(\frac{\pi}{2} \cos \theta\right)}{\sin ^{2}\left(\frac{\pi}{2} \cos \theta\right)}$

With (12), this becomes

$$
R_{0} \mathrm{e}=60(0.945)^{2} \int_{0}^{\pi} \frac{\sin ^{2} n\left(\frac{\pi}{2} \cos \theta\right)}{\sin ^{2}\left(\frac{\pi}{2} \cos \theta\right)} \cdot \sin ^{3} \theta \cdot d \theta
$$

In order to solve this integral, it is convenient to expand

$$
\begin{gathered}
\frac{\sin ^{2} n y}{\sin ^{2} y}: \\
\frac{\sin ^{2} n y}{\sin ^{2} y}=n+\sum_{r=2}^{2 n-2}(2 n-r) \cos r y
\end{gathered}
$$

where $y=(\pi / 2) \cos \theta$ and $r=2,4,6,8 \ldots$ even in teger. With (30), the expression for radiation resistance takes the form:

$$
\begin{aligned}
R_{0} e & =60(0.945)^{2}\left\{n \int_{0}^{\pi} \sin ^{3} \theta d \theta\right. \\
& \left.+\sum_{r=1}^{2 n-2}(2 n-r) \int_{0}^{\pi} \cos \left(r \frac{\pi}{2} \cos \theta\right) \sin ^{3} \theta \cdot d \theta\right] .
\end{aligned}
$$

The first integral is immediately integrable. It is

$$
\int_{0}^{\pi} \sin ^{3} \theta d \theta=\frac{4}{8}
$$

The second integral can be evaluated by Hankel's integral theorem:

$$
\int_{0}^{\pi} \cos (Z \cos \theta) \cdot \sin ^{2} v \theta \cdot d \theta=\frac{J_{\nu}(Z) \Gamma\left(\frac{1}{2}\right) \Gamma\left(\nu+\frac{1}{2}\right)}{\left(\frac{1}{2} Z\right)}
$$

This evaluation holds for $\nu>-\frac{1}{2}$. With $2 \nu=3$ and $Z=r(\pi / 2)$, and recalling that the gamma functions $\Gamma\left(\frac{1}{2}\right)$ and $\Gamma\left(3 / 2+\frac{1}{2}\right)$ have the values $\sqrt{\pi}$ and 1 , respectively, (33) becomes

$$
\begin{aligned}
\int_{0}^{\pi} \cos \left(r \frac{\pi}{2} \cos \theta\right) \sin ^{3} \theta \cdot d \theta & \frac{J_{3 / 2}\left(r \frac{\pi}{2}\right) \cdot \sqrt{\pi} \cdot 2 \sqrt{2}}{\left(r \frac{\pi}{2}\right)^{3 / 2}} .
\end{aligned}
$$

It is well known that

$$
\frac{J_{3 / 2}\left(r \frac{\pi}{2}\right)}{\left(r \frac{\pi}{2}\right)^{3 / 2}}=\sqrt{\frac{2}{\pi}}\left\{\frac{\sin \left(r \frac{\pi}{2}\right)}{\left(r \frac{\pi}{2}\right)^{3}}-\frac{\cos \left(r \frac{\pi}{2}\right)}{\left(r \frac{\pi}{2}\right)^{2}}\right\}
$$

Since $r$ is always an even integer, $\sin [r(\pi / 2)]=0$, and

$$
\frac{J_{3 / 2}\left(r \frac{\pi}{2}\right)}{\left(r \frac{\pi}{2}\right)^{3 / 2}}=-\sqrt{\frac{2}{\pi}} \frac{\cos \left(r \frac{\pi}{2}\right)}{\left(r \frac{\pi}{2}\right)^{2}} .
$$

Hence,

$$
\int_{0}^{\pi} \cos \left(r \frac{\pi}{2} \cos \theta\right) \sin ^{3} \theta \cdot d \theta=-4 \frac{\cos \left(r \frac{\pi}{2}\right)}{\left(r \frac{\pi}{2}\right)^{2}}
$$

If this result is substituted in (31) with (32), the formula for $R_{0}{ }^{e}$ in ohms is obtained:

$$
R_{0}{ }^{e}=60(0.945)^{2}\left\{\frac{4 n}{3}-4 \sum_{r=2}^{2 n-2}(2 n-r) \frac{\cos \frac{r \pi}{2}}{\left(\frac{r \pi}{2}\right)^{2}}\right\} .
$$

For purposes of numerical calculation this formula is much simpler than (1) previously derived by BontschBruewitsch. The results are compared in Table II.

\section{Calculation of the Radiation Resistance of a Broadside Array}

The general radiation resistance formula

$$
R_{0} e^{e}=\frac{60}{2 \pi}(0.945)^{2}\left[\frac{8 \pi n}{3}+4 \pi \sum_{r=2}^{2 n-2}(2 n-r) \cos \left(r K_{2}\right) \Lambda\left(r \pi \frac{s}{\lambda}\right)\right]
$$


gives the radiation resistance of any parallel array consisting of center-fed half-wave dipoles, provided the spacing $s$ between the elements is uniform and the phasing $K_{2}$ is uniformly progressive or zero.

The radiation resistance of a broadside array, $\left[s=(\lambda / 2), K_{2}=0\right]$ is, therefore, given by the specialized case of (26):

$$
R_{0}{ }^{e}=\frac{60}{2 \pi}(0.945)^{2}\left[\frac{8 \pi n}{3}+4 \pi \sum_{r=2}^{2 n-2}(2 n-r) \Lambda\left(r \frac{\pi}{2}\right)\right] .
$$

$R_{0}{ }^{e}=\frac{60}{2 \pi}(0.945)^{2}\left[\frac{8 \pi n}{3}-8 \pi \sum_{r=2}^{2 n-2}(2 n-r) \Lambda\left(r \frac{\pi}{2}\right)\right]$.

The average, radiation resistances $\left(R_{0}{ }^{\circ} / n\right)$ are computed for $n=1,2,3,5,6,7$ and shown in Table II.

In addition, a general formula (26) for the radiation resistance of any uniformly spaced $(s)$ parallel array of half-wave dipoles with uniform progressive phasing $\left(K_{2}\right)$, including zero, is derived.

$$
R_{0}^{e}=\frac{60}{2 \pi}(0.945)^{2}\left[\frac{8 \pi n}{3}+4 \pi \sum_{r=2}^{2 n-2}(2 n-r) \cos \left(r K_{2}\right) \Lambda\left(r \pi \frac{s}{\lambda}\right)\right]
$$

The radiation resistances of broadside arrays consisting of $2,3,4,5,6$, and 7 elements are computed and shown in Table IV.

TABLE IV

Average Radiation Resistance* of Broadside Arrays

\begin{tabular}{clll}
\hline $\begin{array}{c}\text { Number of } \\
\text { elements }\end{array}$ & $P$ & $B B$ & $P K$ \\
\hline$n=2$ & 60.9 ohms & 60.58 ohms \\
3 & 59.5 & 58.78 \\
4 & 58.0 & & 57.27 \\
5 & 57.4 & 56.63 \\
6 & 56.9 & & 56.07 \\
7 & 56.5 & 56 ohms & 55.75 \\
Infinite & &
\end{tabular}

* Average radiation resistance means the total radiation resistance divided by the number of elements: $\left(R_{0}{ }^{\circ} / n\right)$.

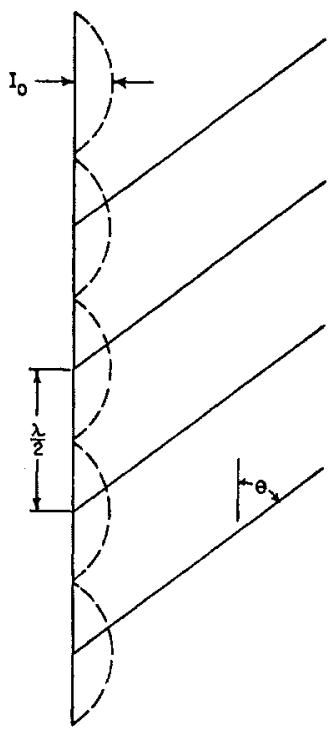

Fig. 1-Collinear array. Radiation resistance for $n$-element collinear array:

$$
R_{0}{ }^{\circ}=60(0.945)^{2}\left\{\frac{4 n}{3}-4 \sum_{r=2}^{2 n-2}(2 n-r) \frac{\cos \left(r \frac{\pi}{2}\right)}{\left(\frac{r \pi}{2}\right)^{2}}\right\} .
$$

\section{Conclusions}

A formula (38) is derived for the radiation resistance of a collinear array consisting of $n$ in-phase half-wave dipoles spaced one half wavelength between centers (Fig. 1). where

$$
\begin{aligned}
\Lambda\left(r \pi \frac{s}{\lambda}\right)= & \frac{\sin \left(r \pi \frac{s}{\lambda}\right)}{\left(r \pi \frac{s}{\lambda}\right)}-\frac{\sin \left(r \pi \frac{s}{\lambda}\right)}{\left(r \pi \frac{s}{\lambda}\right)^{3}} \\
& +\frac{\cos \left(r \pi \frac{s}{\lambda}\right)}{\left(r \pi \frac{s}{\lambda}\right)^{2}} .
\end{aligned}
$$

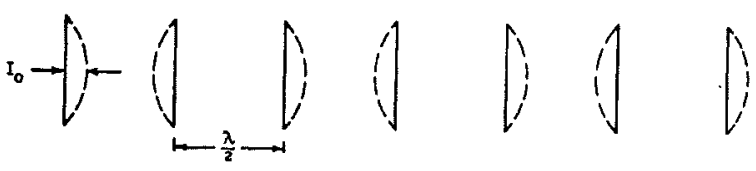

Fig. 2-Broadside end-fire. Radiation resistance for $n$-element broadside array:

$$
R_{0}{ }^{e}=\frac{60}{2 \pi}(0.945)^{2}\left[\frac{8 \pi n}{3}+4 \pi \sum_{1=2}^{2 n-2}(2 n-r) \Lambda\left(r \frac{\pi}{2}\right)\right]
$$

and

$$
\Lambda\left(r \frac{\pi}{2}\right) \equiv \frac{\cos \left(r-\frac{\pi}{2}\right)}{\left(r \frac{\pi}{2}\right)^{2}}
$$

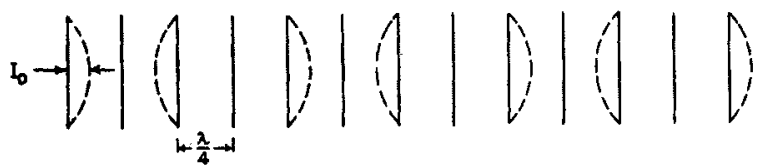

Fig. 3-Unilateral end-fire. Radiation resistance for $n$-element unilateral array:

$$
R_{0^{\circ}}=\frac{60}{2 \pi}(0.945)^{2}\left[\frac{8 \pi n}{3}+4 \pi \sum_{r=2}^{2 n-2}(2 n-r) \cos \left(r \frac{\pi}{4}\right) \Lambda\left(r \frac{\pi}{4}\right)\right]
$$

and

$$
\Lambda\left(r \frac{\pi}{4}\right) \equiv \frac{\sin \left(r \frac{\pi}{4}\right)}{\left(r \frac{\pi}{4}\right)}-\frac{\sin \left(r \frac{\pi}{4}\right)}{\left(r \frac{\pi}{4}\right)^{3}}+\frac{\cos \left(r \frac{\pi}{4}\right)}{\left(r \frac{\pi}{4}\right)^{2}}
$$

7 This equation equals (38) found in an earlier part of this paper. It has been written in this form to place in evidence its similarity to (26). 
By giving $K_{2}$ and $s$ appropriate values, the average radiation resistances of broadside, unilateral end-fire,

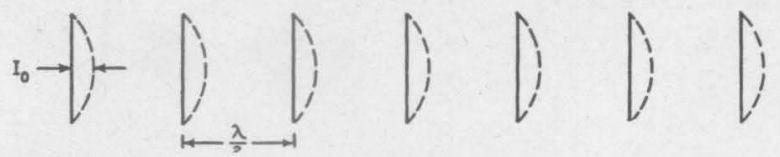

Fig. 4-Bilateral end-fire. Radiation resistance for $n$-element bilateral array:

$$
R_{0^{*}}=\frac{60}{2 \pi}(0.945)^{2}\left[\frac{8 \pi n}{3}+4 \pi \sum_{r=2}^{2 n-2}(2 n-r) \cos \left(r \frac{\pi}{2}\right) \Lambda\left(r \frac{\pi}{2}\right)\right]
$$

and

$$
\Lambda\left(r \frac{\pi}{2}\right) \equiv \frac{\cos \left(r \frac{\pi}{2}\right)}{\left(r \frac{\pi}{2}\right)^{2}}
$$

and bilateral end-fire arrays with $2,3,4,5,6$, and 7 elements (Figs. 2, 3, 4) are computed and shown in Tables I, III, and IV.
The values of $K_{2}, s$, and $\Lambda$ used in the calculation are shown in Table $\mathrm{V}$.

\begin{tabular}{|c|c|c|c|c|}
\hline$K_{2}{ }^{*}$ & $s$ & $\Lambda$ & $\begin{array}{l}\text { Phase } \\
\text { Difference } \\
\text { in Periods }\end{array}$ & $\begin{array}{c}\text { Nature of } \\
\text { Pattern }\end{array}$ \\
\hline $\begin{array}{l}\pi / 2 \\
\pi / 4 \\
0\end{array}$ & $\begin{array}{l}\lambda / 2 \\
\lambda / 4 \\
\lambda / 2\end{array}$ & $\begin{array}{l}\Lambda[r(\pi / 2)] \\
\Lambda[r(\pi / 2)] \\
\Lambda[r(\pi / 2)]\end{array}$ & $\begin{array}{l}\frac{1}{2} \\
\frac{1}{4} \\
0\end{array}$ & $\begin{array}{l}\text { Bilateral end-fire } \\
\text { Unilateral end-fire } \\
\text { Broadside }\end{array}$ \\
\hline$r$ & & $\Lambda[r(\pi / 4)]$ & & $\Lambda[r(\pi / 2)]$ \\
\hline $\begin{array}{r}2 \\
4 \\
6 \\
8 \\
10 \\
12\end{array}$ & & $\begin{array}{c}0.3786 \\
-0.1013 \\
-0.2026 \\
0.02533 \\
0.1252 \\
-0.01126\end{array}$ & & $\begin{array}{l}-0.1013 \\
+0.02533 \\
-0.01126 \\
+0.006333 \\
-0.004053 \\
+0.002814\end{array}$ \\
\hline
\end{tabular}

TABLE V

* $K_{2}$, according to (6), equals $\pi$ times phase displacement measured in fractions of a period.

\section{ACKNOWLEDGMENT}

The authors acknowledge the aid of Miss M. L. Richardson, who performed all the calculations.

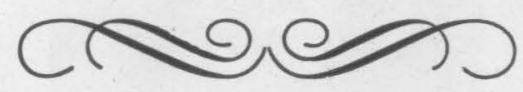

\section{Contributors to the Proceedings of the I.R.E.}

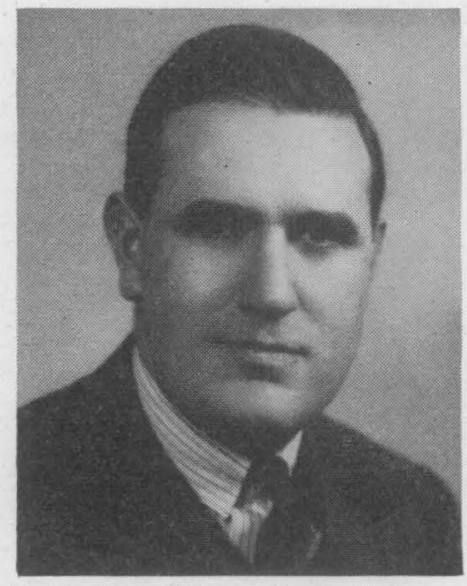

Wanamaker-Underwood and Underwood

Frank J. Bingley

Frank J. Bingley (A'34-M'36-SM'43) was born on November 13,1906, in Bedford, England. Following his graduation from the University of London in 1927, Mr. Bingley was associated with Baird Television Ltd., London, and for two years following he was in charge of Baird's New York Laboratories, after which he joined the Philco Corporation as a television research engineer. $\mathrm{He}$ is now chief television engineer of Philco.

Under Mr. Bingley's direction, television station WPTZ at Wyndmoor, Pa., was designed and constructed in 1941. Mr. Bingley was instrumental in developing the Plane-OScope, a flat-surface picture tube which presents an undistorted picture, regardless of the angle from which it is viewed. Remotepickup equipments in the Philadelphia area, a mobile unit for "on-the-spot" television shows, and the Philco radio relay for television between New York and Philadelphia are other developments instituted by $\mathrm{Mr}$. Bingley.

Mr. Bingley is a director of the Television Broadcasters Association, and a member of the Franklin Institute. He has served as a member of several prominent television planning committees, notably those of RMA, the National Television Systems Committee, and the Radio Technical Planning Board. He is also a member of the Papers Review and Color Television Committees of I.R.E.

Christopher P. Gadsden (M'45) was born in Charlotte, N. C., in 1920 . He received the B.S. degree in electrical engineering from Tulane University in 1942. Immediately thereafter he joined the staff of the Radiation Laboratory at the Massachusetts Institute of Technology, where he remained through 1945. There he worked with the receiver group on the measurement and improvement of the noise figure of intermediate-frequency amplifiers. At the present time he is studying and teaching mathematics at Tulane University.

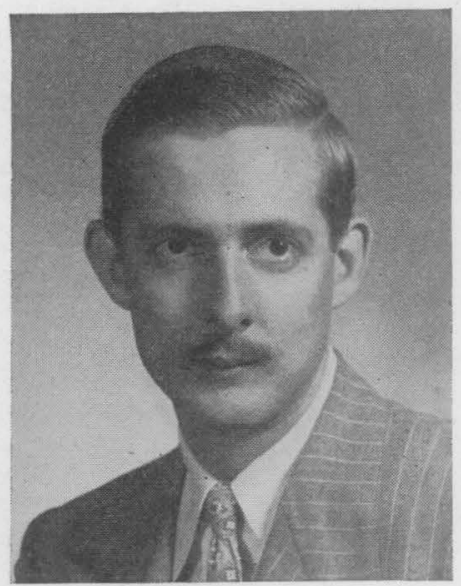

Christopher P. Gadsden

$*$

For a photograph and biography of Ronold KING, see page 244 of the February, 1948 , issue of the Procendings OF THE I.R.E.

$$
*
$$

James E. Hacke, Jr., was born in 1920 at Essex, Iowa. He received the A.B. and M.S. degrees at the University of Georgia in 1940 and 1941, and has done graduate work in physics at Columbia University and The Pennsylvania State College.

From 1943 to $1946 \mathrm{Mr}$. Hacke worked in 